psychopraxis. neuropraxis $2017 \cdot 20: 245-247$ https://doi.org/10.1007/s00739-017-0427-1 Online publiziert: 7. November 2017 (c) Der/die Autor(en) 2017. Dieser Artikel ist eine Open-Access-Publikation.

CrossMark

\title{
Gerhard Ransmayr
}

Klinik für Neurologie 2, Kepler Universitätsklinikum, Med Campus III, Linz, Österreich

\section{Erfolgreiche Behandlung der Fluktuationen und Dyskinesien beim Parkinsonsyndrom}

\section{Therapie mittels intrajejunal intestinalem Gel}

\section{Einleitung}

Die Behandlung mit kontinuierlich intrajejunal und intestinal appliziertem Levodopa-Carbidopa-Gel (LCIG) bei Patienten mit fortgeschrittener ParkinsonKrankheit ist seit mehreren Jahren etabliert. Vor 3 Jahren [1] wurde die erste große multizentrische kontrollierte randomisierte doppelblinde Studie zu dieser Therapie veröffentlicht. Sie zeigte eine deutliche Verbesserung von Patienten, die diese Therapie erhielten, vor allem im Hinblick auf eine Verminderung der Zeit schlechter Beweglichkeit (Off-Phasen).

\section{Fallbeispiel}

An der Klinik für Neurologie II des Med Campus III des Kepler Universitätsklinikums wurde 2010 eine Patientin mit 9-jähriger Parkinson-Erkrankungsdauer vorstellig. Sie litt unter starken motorischen Fluktuationen und Dyskinesien. Die Versuche einer diesbezüglichen oralen Therapieoptimierung blieben erfolglos. Die Patientin sprach aber im L-Dopa-Test exzellent an, war in einer ausgezeichneten psychischen Verfassung, litt subjektiv unter keinerlei kognitiven Einschränkungen, hatte ein gutes Gedächtnis (Cerad-plus), leichte visuell-räumliche Schwierigkeiten und war aufgrund der starken motorischen Fluktuationen stundenlang bei Tag und auch nachts schlecht beweglich. In Phasen guter Beweglichkeit litt sie unter starken Dyskinesien.
Die Patientin war auch an einer Hypertonie erkrankt, an einer Atherosklerose, einem chronischen Wirbelsäulenleiden (degenerative Veränderungen sowie Zustand nach Spondylodiszitis mit Spinalkanalstenose). Beidseitig war sie an den Hüften wegen Koxarthrose operiert und an der Wirbelsäule wegen eines Bandscheibenvorfalls sowie einer Wirbelkanalengstelle. Aufgrund einer langjährigen Levodopa-Therapie in Kombination mit Pramipexol retard bestand ein relativer Vitamin-B12-Mangel. Außerdem litt sie an einer Osteoporose sowie an einer motorischen, gering sensorischen, distal akzentuierten Polyneuropathie.

)> Die LCIG-Therapie wird für Patienten im fortgeschrittenen Stadium empfohlen

Zuletzt wurde die dopaminerge Therapie so angepasst, dass die Patientin zwar nicht unter starken Dyskinesien litt, jedoch im schlimmsten Fall bis zu $50 \%$ der Wachzeit an deutlich verminderter Beweglichkeit. Durch plötzliches Auftreten des „freezing of gait" war die Patientin erheblich sturzgefährdet. Sie wurde ausführlich über die Möglichkeit einer Pumpenbehandlung mittels kontinuierlicher LCIG-Infusion informiert und willigte nach mündlicher und schriftlicher Aufklärung über den Eingriff (Gastroskopie, Anlegen einer PEG-Sonde mit Katheter bis in das Jejunum) ein. 
Hier steht eine Anzeige. 黛 Springer psychopraxis. neuropraxis https://doi.org/10.1007/s00739-017-0427-1

(c) Der/die Autor(en) 2017. Dieser Artikel ist eine Open-Access-Publikation.

\section{G. Ransmayr}

\section{Erfolgreiche Behandlung der Fluktuationen und Dyskinesien beim Parkinsonsyndrom. Therapie mittels intrajejunal intestinalem Gel}

\section{Zusammenfassung}

Für Parkinson-Patienten im fortgeschrittenen Erkrankungsstadium stellt die Infusion eines Levodopa-Gels in den Dünndarm seit mehreren Jahren eine Alternative zur oralen Therapie dar. Patienten, die lange Zeit unter einer Medikation stehen, entwickeln häufig motorische Fluktuationen und

Dyskinesien. Im beschriebenen Fallbeispiel erhielt die Patientin im Alter von 70 Jahren die Levodopa-Carbidopa-Intestinal-Gel-
Therapie (LCIG) und findet auch mehr als drei Jahre nach Beginn der Therapie, dass ein eindeutiger Nutzen im Sinne einer Abnahme der Phasen schlechter Beweglichkeit (OffPhasen) über $50 \%$ des Tages besteht.

\section{Schlüsselwörter}

Morbus Parkinson - Levodopa-Carbidopa instestinales Gel · Dyskinesien · Motorische Fluktationen · Fallbericht

\section{Successful treatment of fluctuations and dyskinesias in Parkinson syndrome. Intrajejunal infusion of intestinal gel}

\section{Abstract}

For patients with advanced Parkinson's disease, the infusion of a levodopa gel into the small intestine has been an alternative to oral therapy for several years. Patients who are under long-term medication frequently develop motor fluctuations and dyskinesias. Here, we describe the case of a patient received the levodopa-carbidopa intestinal gel (LCIG) therapy at the age of 70 years.
Since having started therapy three years ago, she continues to report a clear benefit in the sense of a $50 \%$ decrease in the daily number of phases of poor mobility (off phases).

\section{Keywords}

Parkinson disease - Levodopa-carbidopa intestinal gel - Dyskinesia - Motor fluctuations . Case report

\section{Vor dem Eingriff}

Zum Zeitpunkt des Eingriffs litt die Patientin unter hohen Blutdruckwerten, war aber ansonsten in einem guten körperlichen Zustand. Sie nahm Levodopa-Carbidopa-Entacapone $4 \times$ 150/37,5/200 mg ein, sowie Pramipexol retard $0,52 \mathrm{mg}$ abends. Schließlich erhielt die Patientin wegen Dyskinesien morgens und mittags eine Tablette Amantadinsulfat $100 \mathrm{mg}$. Zur Besserung des Nachtschlafs und auch zur Verbesserung der Beweglichkeit nahm sie abends eine Kapsel 100/25 mg retardiertes Levodopa ein. Wegen wiederkehrender Gastritis nahm sie Pantoprazol, wegen einer Osteoporose Kalzium und Vitamin D3. Eine mehrtägige Analyse mittels Tageskalender, wobei die Patientin beim Ausfüllen vom Pflegepersonal unterstützt wurde, ergaben OFF-Phasen, die im schlimmsten Fall $50 \%$ der Tageswachphase betrafen.
Die Patientin wurde vor dem eigentlichen Eingriff noch mit einer Naso-Duodenal-Sonde versorgt, um ihr auf diese Art und Weise eine Entscheidungshilfe für die LCIG-Therapie zu geben und um ihr zu zeigen, dass die LCIG-Therapie wirkt. Die Sonde konnte ohne $\mathrm{Ne}$ benwirkungen und Komplikationen dauerhaft eingesetzt werden. In den darauf folgenden Tagen wurde die Patientin mit LCIG-Infusion von 5-8 $\mathrm{ml}$ pro Stunde (als Morgendosis) behandelt, die kontinuierliche Tagesdosis betrug zwischen 2 und 2,8 $\mathrm{ml}$ pro Stunde, Einzeldosen (Bolus zur Verbesserung der Beweglichkeit) wurde 2-4 $\times$ täglich zusätzlich in einer Dosis von $0,7-1,5 \mathrm{ml}$ verabreicht.

\section{Nach dem Eingriff}

In den postoperativen Tagen konnte bei Adjustierung der LCIG-Dosis die orale Therapie deutlich reduziert werden. So waren zuletzt als orale The- 
rapie nur mehr L-Dopa-CR 100/25 mg 2 Stück um 22 Uhr (zum Zeitpunkt der Abnahme der LCIG-Pumpe) erforderlich. Gegen eine sich in den letzten Jahren entwickelnde sensomotorische Polyneuropathie bei Vitamin-B12-Mangel (laborchemischer Nachweis) erhielt die Patientin ein Vitamin-B-Kombinationspräparat $3 \times$ täglich. Ansonsten nahm sie die vorbestehende internistische Therapie mit Valsartan, Furosemid, Kaliumchlorid, Magnesiumcitrat sowie zur Schmerzbehandlung Lornoxicam $8 \mathrm{mg}$. Die Behandlung der chronischen Gastritis mit Pantoprazol $40 \mathrm{mg}$ ging unverändert weiter.

\section{Schlussfolgerung}

Trotz Beeinträchtigung der Beweglichkeit durch schwere Residualveränderungen der Lendenwirbelsäule und einer Polyarthrose sowie Zustand nach Hüftgelenksersatz beidseits besserte sich die Mobilität der Patientin beträchtlich. Sie war durchwegs, in erster Linie reaktiv auf den Therapieerfolg, gut gestimmt. Diese Besserung hält 3 Jahre nach Beginn dieser Therapie an. Wegen einer Doppelschlingenbildung der Jejunalsonde musste diese vor wenigen Monaten ersetzt werden. Ansonsten bestanden keine Therapiekomplikationen. Die Patientin wird in 3- bis 6-monatigen Abständen vonseiten des Pflegeteams der Firma und von einer Fachärztin für Neurologie kontrolliert. Die Pumpe ist ein wenig bei Bewegungen und bei Alltagsverrichtungen hinderlich, der therapeutische Effekt jedoch sehr zufriedenstellend und der Zustand eindeutig besser als vor Implementierung der LCIG-Therapie. Die Off-Zeiten reduzierten sich auf maximal 1,5 Stunden pro Tag. Bis dato wurden keinerlei kognitive oder psychiatrische Nebenwirkungen beobachtet.

\section{Fazit für die Praxis}

\footnotetext{
- Die Kombination aus den Wirkstoffen Levodopa und Carbidopa wird bei Patienten mit fortgeschrittener Parkinson-Krankheit, insbesondere zur Behandlung sogenannter OFFPhänomene eingesetzt um damit die ON-Phasen zu verlängern.
}

\section{- Die typischen Bewegungsstörungen wie stockende Beweglichkeit, Mus- kelstarre oder Zittern werden durch eine langfristige Einnahme erheblich verringert.}

\section{Korrespondenzadresse}

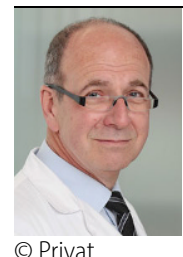

Prim. Univ.-Prof. Dr.

G. Ransmayr

Klinik für Neurologie 2, Kepler Universitätsklinikum, Med

Campus III

Krankenhausstraße 9,

4021 Linz, Österreich gerhard.ransmayr@ kepleruniklinikum.at

Funding. Open access funding provided by Johannes Kepler University Linz.

\section{Einhaltung ethischer Richtlinien}

Interessenkonflikt. G. Ransmayr gibt an für Beratungstätigkeiten, Vorträge und Kongressreiseunterstützung Honorare von Abbvie, Grünenthal Ges.m.b.H. Österreich, Krka Pharma, Novartis und Ratiopharm erhalten zu haben.

Dieser Beitrag beinhaltet keine von den Autoren durchgeführten Studien an Menschen oder Tieren.

Open Access Dieser Artikel wird unter der Creative Commons Namensnennung 4.0 International Lizenz (http://creativecommons.org/licenses/by/4.0/deed de) veröffentlicht, welche die Nutzung, Vervielfältigung, Bearbeitung, Verbreitung und Wiedergabe in jeglichem Medium und Format erlaubt, sofern Sie den/die ursprünglichen Autor(en) und die Quelle ordnungsgemäßnennen, einen Linkzur Creative Commons Lizenz beifügen und angeben, ob Änderungen vorgenommen wurden.

\section{Literatur}

1. Olanow CW et al (2014) Continuous intrajejunal infusion of levodopa-carbidopa intestinal gel for patients with advanced Parkinson's disease: a randomised, controlled, double-blind, doubledummy study. Lancet Neurol 13(2):141-149. https://doi.org/10.1016/S1474-4422(13)70293-X
Hier steht eine Anzeige.

\section{Springer}

